\title{
TRSS: a new version of program TRS for a different geometry
}

\author{
Joachim Schmitz ${ }^{1}$, Hans-Rainer Trebin ${ }^{2}$ \\ Institut für Theoretische und Angewandte Physik, Universität Stuttgart, Pfaffenwaldring 57, W-7000 Stuttgart 80, Germany
}

and

\author{
Ulrich Rössler ${ }^{3}$ \\ Institut für Theoretische Physik, Universität Regensburg, Universitätsstrasse 31, W-8400 Regensburg, Germany
}

Received 19 July 1991

\begin{abstract}
Quantum resonances in the bands of semiconductors under uniaxial stress provide very detailed information on the band parameters. However, the analysis of experimental data is difficult. Computer programs based on an adequate theoretical model make this task easier. Program TRSS calculates energy eigenvalues, wave functions and oscillator strengths for direct inter- and intraband dipole transitions. The magnetic field is applied parallel to the [001] crystal axis while the uniaxial stress is directed perpendicular [100] to it.
\end{abstract}

\section{NEW VERSION SUMMARY}

Title of new version: TRSS

Catalogue number: ACGS

Program obtainable from: CPC Program Library, Queen's University of Belfast, N. Ireland (see application form in this issue)

Reference to original program: Comput. Phys. Commun. 66 (1991) 308; catalogue number: ACBH

Authors of original program: J. Schmitz, H.-R. Trebin and U. Rössler

Does the new version supersede the original program? no

Licensing provisions: none

Computer: VAX II/GPX; Installation: Institut für Theoretische und Angewandte Physik, Universität Stuttgart, Pfaffenwaldring 57 , W-7000 Stuttgart 80 , Germany

Operating system under which the new version has been tested: VAX/VMS 4.6

1 joa $@$ ds0ita51.bitnet

2 trebin@ds0ita51.bitnet

3 roessler@vax1.rz.uni-regensburg.dbp.de
Programming language used in the new version: standard FORTRAN 77

Memory required to execute with typical data: 434 Kbytes

No. of bits in a word: 32

Peripherals used: terminal, disk

No. of lines in distributed program, including test data, etc: 5207

Separate documentation available: TRS User's and Programmer's Guide: no. of pages: 76: available from: CPC Program Library, or Joachim Schmitz, Institut für Theoretische und Angewandte Physik, Universität Stuttgart, Pfaffenwaldring 57, W-7000 Stuttgart 80, Germany, joa@ds0ita51.bitnet

Keywords: narrow-gap semiconductors, zincblende lattice, Landau levels, uniaxially stress parallel to magnetic field, effective Hamiltonian, invariant expansion, eightfold space of valence band and lowest conduction band, normal and inverted bands, energy eigenvalues, eigenvectors, direct interand intraband dipole transitions, oscillator strengths, $\Gamma$-point, [001] and [100] crystal axis, uniaxial stress perpendicular to magnetic field 


\section{Nature of physical problem}

An effective Hamiltonian constructed by invariant expansion is used to calculate Landau levels and wave functions in narrow-gap semiconductors with a zincblende or diamond lattice under uniaxial stress [4]. It is based on an eightfold space of uppermost valence and lowest conduction bands at the center of the Brillouin zone and its vicinity. The wave functions are further used to calculate the oscillator strengths of direct inter- and intraband dipole transitions. Thus the TRSS program is a valuable tool for the experimentalist to analyze quantum resonances measured in semiconductors.

\section{Method of solution}

The matrix elements of the Hamiltonian are set up one by one according to the equations derived from the theory [2,3]. Then the resulting matrix is diagonalized using I Iousebolder's reduction followed by the QL method. Energy eigenvalues and eigenvectors are further used in calculation of oscillator strengths.

Reasons for the new erision

Adaptation for a different geometry

Restrictions on the complexity of the problem

The dimensions of arrays are set to include Landau levels with oscillator quantum number up to $n_{u} \leq 39$. Adaptations are easily made. Due to the limitations in the Kane-modell [1] and the underlying perturbation theory the program is only suitable for eigenstates in the vicinity of the $I$-point. Transitions are restricted to direct dipole transitions. All calculat tions are based on a geometry with magnetic field parallel to the $[(0) 1]$ crystal axis and uniaxial stress applied parallel to the [100] crystal axis

Typical runing time

Same as for program TRS

Unusual features of the program

TRSS contains a subroutine which clears the screen of the terminal before displaying a new page of text. This action is not essential to the operation of the program and may be entirely omitted. In order to preserve the intended screen display it must be adapted to the specific device used.

References

11] E.O. Kane. in: Semiconductors and Semimetalls. vol. I. eds. R.K. Willardson and A.C. Beer (Academic. New York, 1966$)$ p. 75.

[2] H.-R. Trebin, U. Rössler and R. Ranvaud. Phys. Rev. B 2() (1979) 686 .

[3] H.-R. Trebin, W. Wolfstädter. H. Pascher and H, Häfele. Phys. Rev. B 37 (1988) 10249.

[4] G.L. Bir and G.E. Pikus. Symmetry and Strain-Induced Effects in Semiconductors (Wiley, New York, 1974).

\section{LONG WRITE-UP}

Detailed information on the band parameters of a semiconductor can be obtained from quantum resonances in uniaxially stressed crystals. To this end the experimental data must be analyzed on the basis of a theoretical model. This model was provided by Trebin and Rössler [1,2] for narrow-gap semiconductors with a zincblende lattice. It is based on an effective Hamiltonian constructed by invariant expansion. A concise description of the theory has already been given in ref. [3].

The secular problem posed by this Hamiltonian may be solved for any geometry, i.e. with no regard for the directions of magnetic field and uniaxial stress relative to the crystal axes. However, in experiments only a few sclected configurations are used corresponding to high-symmetry directions. Incorporating any of these into the equations greatly simplifies the Hamiltonian and the secular problem. Trebin et al. [1] set up the Hamiltonians for magnetic fields parallel to uni- axial stress applied along the [001], [111], and [110] crystal axes. Program TRS [3] is based upon the geometry where both magnetic field and stress are directed parallel to [001].

Later, Trebin et al. [2] showed that under crossed magnetic field and stress the Landau levels of semiconductors are separated much stronger with stress yielding more insight into the band structure than in the parallel configuration. They compared geometries where the magnetic field is directed along the [001] crystal axis and uniaxial stress either parallel or perpendicular [100] to it. Surprisingly, the modifications to the Hamiltonian - though essential - are minor. Moreover, the selection rules for direct inter- and intraband transitions stay the same. Therefore it was possible to adapt program TRS to this new configuration without major changes. The new program is given the name TRSS. Essential adaptations are made in subroutine MATRIX where the matrix Hamiltonian is built up. All other 
adaptations concern text strings of the interactive menu displays and descriptive comments in the FORTRAN code.

Since no further changes are necessary, all input and output data formats remain the same. The separate documentation "TRS User's and Programmer's Guide" is valid throughout for program TRSS as well. The only differences are visible in the interactive menu displays and naturally in the results of the calculations. Two pages from the test run are shown in this paper, the test run input being identical for both TRS and TRSS.

\section{References}

[1] H.-R. Trebin, U. Rössler and R. Ranvaud, Phys. Rev. B 20 (1979) 686.

[2] H.-R. Trebin, W. Wolfstädter, H. Pascher and H. Häfele, Phys. Rev. B 37 (1988) 10249.

[3] J. Schmitz, H.-R. Trebin, and U. Rössler, Comput. Phys. Commun. 66 (1991) 308. 


\section{TEST RUN OUTPUT}

*** LANOAU-LEVELS IN ZINCBLENDE-TYPE SEMICONDUCTORS ***

INSB. DAT

REPRESENTATION 2 - eigenvalues and eigenvectors from $28 \mathrm{~V} 14 \mathrm{C}$ 14S functians

Eigenvalues

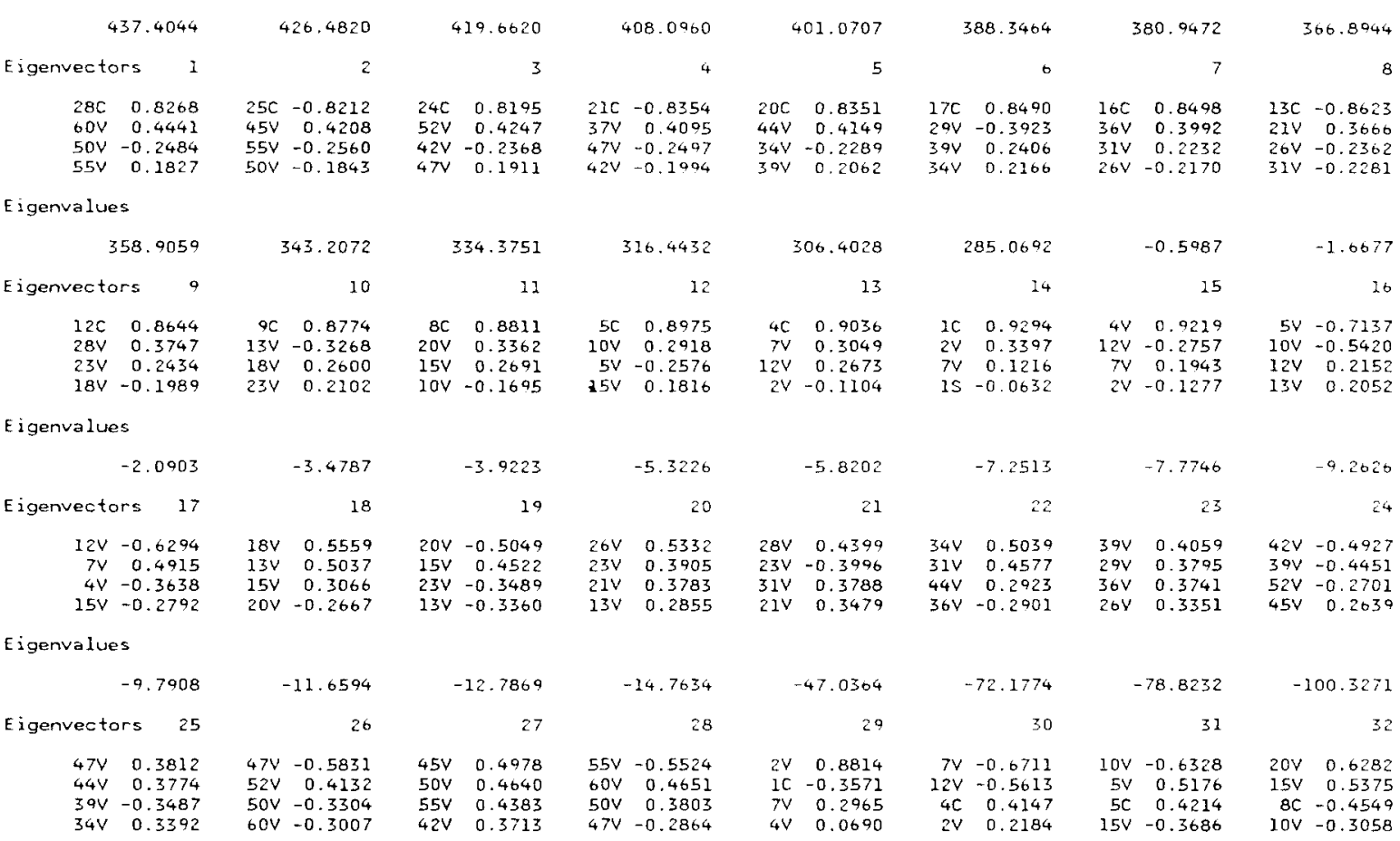

*** LANDAU-LEVELS IN ZINCBLENDE-TYPE SEMICONDUCTORS ***

INSB. DAT

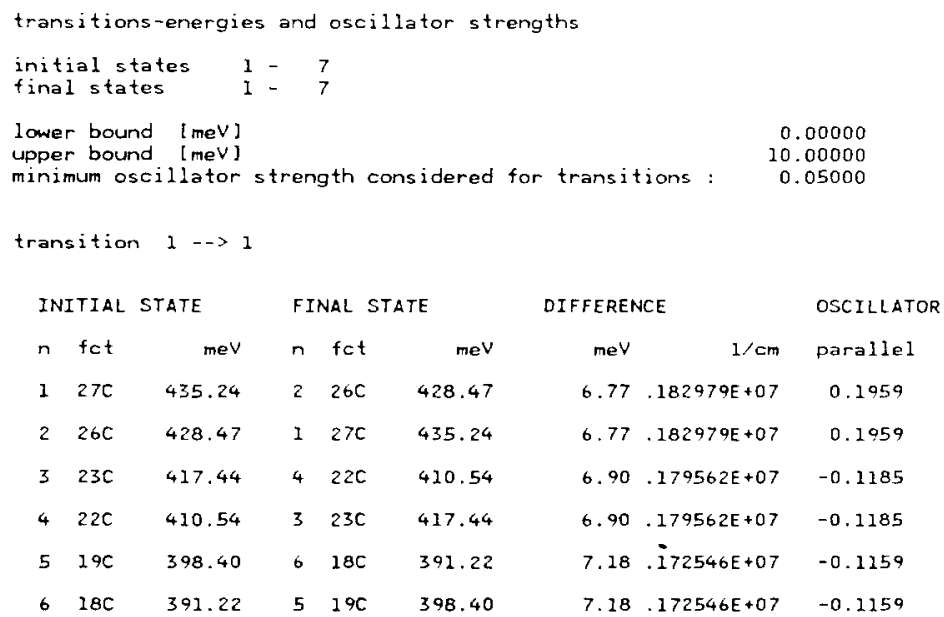

